\title{
Variation of diurnal Turbulence Kinetic Energy using sonic anemometer observation: a case study Ledeng, Bandung
}

\author{
Sandy Hardian Susanto Herho \\ Department of Meteorology, \\ Faculty of Earth Sciences and Technology, \\ Bandung Institute of Technology, \\ Jl. Ganesha 10, Labtek XI, $2^{\text {nd }}$ floor, Bandung, \\ Indonesia 40132 \\ sandyherho@meteo.itb.ac.id
}

\author{
Dasapta Erwin Irawan \\ Applied Geology Research Group, \\ Faculty of Earth Sciences and Technology, \\ Bandung Institute of Technology, \\ J1. Ganesha 10, Labtek IV, $2^{\text {nd }}$ Floor, Bandung, \\ Indonesia 40132 \\ erwin@gc.itb.ac.id
}

\begin{abstract}
Sonic anemometer observation was performed on 29 - 30 September 2014 in Ledeng, Bandung to see diurnal variations of Turbulence Kinetic Energy (TKE) that occurred in this area. The measured sonic anemometer was the wind velocity components $u, v$, and $w$. From the observation result, it can be seen that the diurnal variation that happened was quite significant. The maximum TKE occurs during the daytime when atmospheric conditions tend to be unstable. TKE values was small at night when atmospheric conditions are more stable than during the daytime.
\end{abstract}

Keywords-diurnal variation, boundary layer observation, turbulence kinetic energy

\section{INTRODUCTION}

Turbulence is a movement of random rotation in a relatively short time [3]. Turbulence greatly affects the boundary conditions of an atmosphere because turbulence transfers energy and heat, vertically and affects the stability of a layer. One way to know the energy of a turbulence is to look for a deviation of a velocity component from its average [1]. Using Reynolds average, the velocity component can be divided into mean and perturbation parts. From the perturbation results, we can calculated Turbulence Kinetic Energy ( $T K E)$. TKE is the kinetic energy possessed by a turbulent. By looking at the kinetic energy of turbulence, we can observe the intensity of the turbulence that occurs.

\section{Methodology}

Data used in this research is data from observation of sonic anemometer (Fig. 1). This observation was conducted on 29 September 2014 from 19.00 Indonesia Western Standard Time (IWST) to 30 September 2014 at 19.00 IWST in the area of Indonesia University of Education (UPI), Ledeng, Bandung (Figure 2) at coordinates $6^{\circ} 51$ ' 48 "S, $107^{\circ} 35^{\prime} 40^{\prime \prime} \mathrm{E}$ (Figure 2). Observation of wind velocity components performed every minute with duration of 24 hours. From observation using the sonic anemometer, we get $u, v$, and $w$ wind components data, which will then be used to calculate $T K E$ in the area for 24 hours.

Often happens, conditions that allow surface airwaves to form wind shears locally, resulting in the formation of turbulence [1]. Therefore, the phenomenon of airwaves in the boundary layer can be associated with the propagation of heat turbulence and the spread of pollutants. The turbulence of the boundary layer is generally influenced by diurnal cycle variations, so the turbulence values in daytime and night will be different [3]. Turbulence is also affected by the stability of the atmosphere in the boundary layer. The stability of the atmosphere is the tendency of air to resist vertical movement or to resist the turbulence that occurred [2]. The stability of the atmosphere depends on the temperature gradient. If the temperature is high, a lot of turbulence phenomenon are expected.

Fig. 1. Sonic Anemometer used in this research observation.

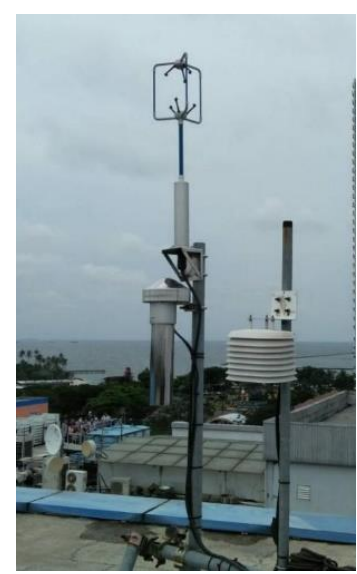


Fig. 2. Google Earth satellite imagery at the research location (marked with red dots).

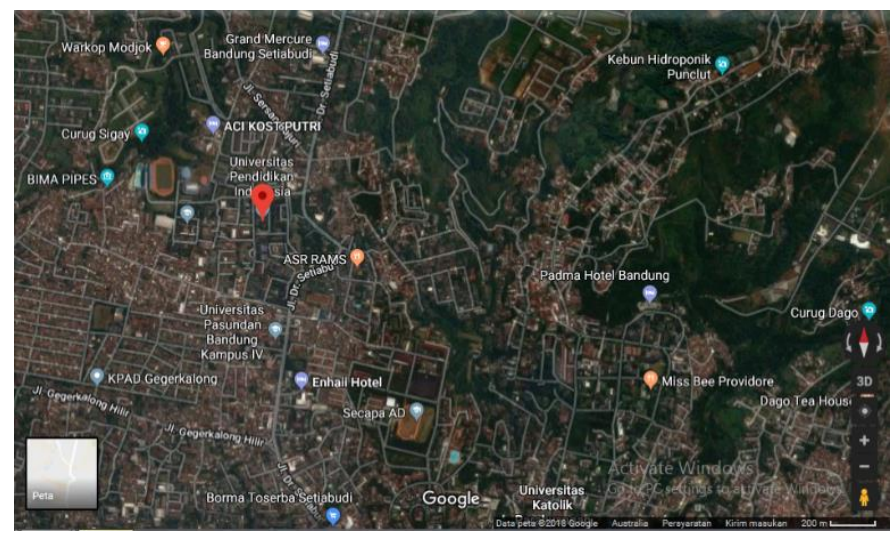

A common approach in studying boundary turbulence is through the calculation of fluid parameters in the boundary layer [3].The boundary layer fluid flow can be divided into mean and turbulent parts [3]. The kinetic energy per unit mass is defined by equation (1).

$$
K=\frac{1}{2} \times\left(u^{2}+v^{2}+w^{2}\right)
$$

, where $u, v$, and $w$ are components of wind velocity.

There is a relatively easy way to simplify fluid motion parameters to measure turbulence in the boundary layer. It is by averaging the observed wind velocity for a time range above 30 minutes to one hour [1]. By averaging the wind velocity, we can eliminate positive and negative deviations from wind velocity (Fig. 3). By using Reynolds averaging, we can separate a stream into its mean and turbulent parts as described in equations (2), (3), and (4).

Fig. 3. The deviation of the wind velocity $u$ from the average velocity. A positive turbulence means that the real wind velocity is greater than the average wind velocity. Turbulence in negative value meaning that the real wind velocity is greater than the average velocity. (modification from Stull, 1988)

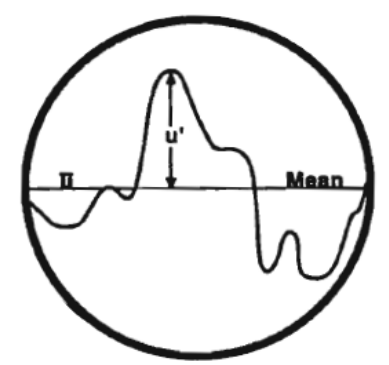

$$
\begin{aligned}
u^{\prime} & =U-\bar{U} \\
v^{\prime} & =V-\bar{V} \\
w^{\prime} & =W-\bar{W}
\end{aligned}
$$

By substituting equations (2), (3), and (4) into equation (1), we can obtain equations (5) and (6) below:

$$
\begin{aligned}
& \frac{M K E}{m}=\frac{1}{2} \times\left(\bar{U}^{2}+\bar{V}^{2}+\bar{W}^{2}\right) \\
& \frac{T K E}{m}=\frac{1}{2} \times\left(u^{\prime 2}+v^{\prime 2}+w^{\prime 2}\right)
\end{aligned}
$$

, where $M K E$ represents the kinetic energy of the average stream and $T K E$ represents the kinetic energy of the turbulent flow. $T K E$ can be generated from shear, friction or buoyancy, or by the eddy current. $T K E$ can be reduced if the condition of a layer more stable. TKE can be represented as the intensity of a turbulence event.

\section{RESULT AND DISCUSSION}

In Fig. 4, it appears that $T K E$ reaches its maximum value at 13.00 IWST. The value of TKE began to increase at 12.00 IWST and decreased at 15.00 IWST. This fluctuation occurs because, at 11.00 IWST, mixed layer has begun to form so that the atmosphere becomes well mixed and there are many turbulence phenomena during the daytime. The highest $T K E$ value is obtained at 13.00 IWST when the sun's intensity reaches its peak. One of the main sources that affecting turbulence is the intensity of the sun, so that when the sun's intensity is high, i.e. during the daytime, atmospheric conditions tend to be unstable and turbulence occurs [3]. TKE value at night tends to be lower than at daytime. The cause is, at night the intensity of solar radiation is much less than during the daytime, so the atmosphere condition tends to be more stable and the atmosphere is not too turbulent.

Fig. 4. Time-series data of Turbulence Kinetic Energy (TKE) during one-day observation. It appears that the peak of turbulence occurs during the daytime.

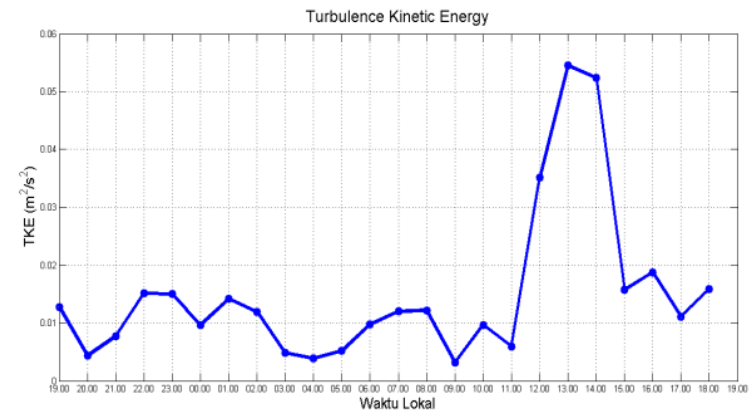

\section{CONSCLUSION}

Through the TKE values, we know the intensity of turbulence phenomena that occur. During the day, the TKE value reaches its maximum point at 13.00 IWST due to unstable atmospheric conditions. At night, atmospheric conditions tend to be more stable than daytime, so the $T K E$ values are much lower. 


\section{ACKNOWLEDGMENT}

We thank I Dewa Gede Agung Junnaedhi GMT and Raden Mas Dwiriyo Suryosasmoko for helpful comments and suggestions.This work was partially funded by Weather and Climate Prediction Laboratory (WCPL) ITB.

\section{REFERENCES}

[1] Dryden, W.A., 1957: EFFECTS OF THE SCALE OF SPATIAL AVERAGING ON THE KINETIC ENERGIES OF SMALL-SCALE TURBULENT MOTION. J. Meteor., 14, 287-292, https://doi.org/10.1175/1520-0469(1957)014<0287:EOTSOS>2.0.CO;2

[2] Holton, J. R. (2004). An Intoduction to Dynamic Meteorology $4^{\text {th }}$ Edition, Elsevier, Burlington.

[3] Stull, R.B., (1988). An Introduction to Boundary Layer Meteorology, Kluwer Academic Publishers, Dordrecht. 